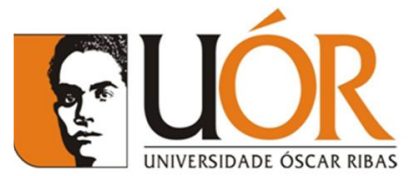

\title{
EL COMERCIO TRADICIONAL EN LA ZONA METROPOLITANA DE GUADALAJARA, MÉXICO. EL CASO DE LOS TIANGUIS
}

Traditional trade in the metropolitan area of Guadalajara, México. The case of swap meets / O comércio tradicional na zona metropolitana de Guadalajara, México: o caso dos tianguis

Victor Castillo ${ }^{1}$ y Suhey Ayala ${ }^{2}$

\section{RESUMO}

O presente artigo enfoca-se no comércio tradicional nos tianguis, entendidos como mercados itinerantes, cujas actividades se realizam ao ar livre, certos dias da semana, num espaço público designado pelas autoridades municipais, com prévio pagamento de uma quota (Delgado, 1992); (Teja e López, 2013). O objectivo é de analisar a importância dos tianguis, no abastecimento e distribuição de produtos básicos da zona metropolitana de Guadalajara, México, incluindo as características distintivas dos actores envolvidos, assim como as prácticas e processos de intercâmbio que eles mobilizam e os resultados associados. Aplicouse um questionário a um grupo tianguistas (382) e de clientes (387), considerados como a uma amostra estatisticamente representativa. Apesar da sua dispersão geográfica, diversidade em tamanho e voltas comerciais, guardam certa semelhança, em termos da informalidade dos processos de trabalho e laxismo, para cumprir com as normas oficiais, particularmente em matéria de segurança e higiene do espaço, mobiliário e equipamento utilizado, assim como nos preços e qualidade dos produtos. O seu desenvolvimento tem sido favorecido pelas vantagens que oferecem para clientes e comerciantes, aumentando a capacidade destes para aproveitar os espaços institucionais. Conclui-se que, apesar da sua importância para o autoemprego, o abastecimento de produtos básicos para um sector da população e para a convivência social, a sua permanencia, deveria referir aspectos associados à qualidade dos serviços, à origem e segurança dos alimentos que alí se vendem e são consumidos.

Palavras chave: Comércio tradicional, tianguis, comércio à retalho, mercado sobre rodas.

Recebido: Dezembro 2017 Aceitado: Janeiro 2018

\section{RESUMEN}

El presente artículo se enfoca al comercio tradicional en los tianguis, entendidos como mercados itinerantes cuyas actividades se realizan al aire libre ciertos días

\footnotetext{
${ }^{1}$ Doctor en Economía Rural. Profesor investigador de la Universidad de Guadalajara. Guadalajara, México. Correo electrónico: victorm.castillog@gmail.com

2 Doctor en Educación. Profesor investigador de la Universidad de Guadalajara. Guadalajara, México. Correo electrónico: suhey.ayala@academicos.udg.mx
} 
de la semana, en un espacio público designado por las autoridades municipales previo pago de una cuota (Delgado, 1992; Teja y López, 2013). El objetivo es analizar la importancia de los tianguis en el abasto y distribución de productos básicos de la zona metropolitana de Guadalajara, México, incluyendo las características distintivas de los actores involucrados, así como las prácticas y procesos de intercambio que ellos movilizan y los resultados asociados. Se aplicó una encuesta a una muestra estadísticamente representativa de tianguistas (382) y de clientes (387). Resultando que, a pesar de su dispersión geográfica y diversidad en tamaño y giros comerciales, guardan cierta similitud en términos de la informalidad de los procesos de trabajo y laxitud para cumplir con las normas oficiales, particularmente en materia de seguridad e higiene del espacio, mobiliario y equipo utilizado, así como en los precios y calidad de los productos. Su desarrollo se ha favorecido por las ventajas que ofrecen para clientes y comerciantes, aunado a la capacidad de estos últimos para aprovechar los huecos institucionales. Se concluye que, a pesar de su importancia para el autoempleo, el abasto de productos básicos para un sector de la población y para la convivencia social, su permanencia debería referir aspectos asociados con la calidad de los servicios y, con el origen y seguridad de los alimentos que allí se venden y se consumen.

Palabras clave: Comercio tradicional, tianguis, comercio minorista, mercado sobre ruedas.

\begin{abstract}
This work was centered on the traditional commerce in the swap meets, which are itinerant markets in the streets some days a week in a public space assigned by the local government once the fee is paid (Delgado, 1992; Teja y López, 2013). The principal objective was to analyze the importance of supply and distribution of goods in the metropolitan area of Guadalajara, Mexico, and so on the exchange process and practice that took place in that spaces. The work is based on data from a survey applied to a sample of tianguistas (382) and customers (387) in which despite the geographic dispersion, size and commercial business diversity, they have a kind of similarities in terms of informality in the working process and laxity to follow the official standards, particularly in security matters, space and furniture hygiene that is required for that kind of commerce. At the same time the prices and quality of the products. It is remarkable that the development has been favored by the advantage that swap meets offer for customers as the same for merchants next to the capability of the merchants to take advantage for the institutional holes. In conclusion, however the importance for self-employment, the acquisitions of basic goods for the population and social interaction, the permanence of the swap meets it has to be associated with the quality of services, and over the less, with the origin and security of the food that are sold and consumed there.
\end{abstract}

Keywords: Traditional trade, Swap meets, Retail, Mobile food market. 
El comercio tradicional en la zona metropolitana de Guadalajara, M éxico. El caso de los tianguis

\section{Introducción}

El tianguis es un mercado itinerante cuyas actividades se realizan al aire libre ciertos días de la semana, conocidos como "días de plaza", en un espacio público designado por las autoridades municipales previo pago de una cuota por metro ocupado por cada uno de los comerciantes, comúnmente conocidos como tianguistas (Delgado, 1992; Bayona, 2011; Teja y López, 2013; García, et, 2016). De acuerdo con Espinosa (2014), los tianguis en México datan de la época prehispánica, aunque a lo largo del tiempo se han transfigurado en sintonía con el proceso de mestizaje característico del país y, por consecuencia, en ellos se mezcla lo tradicional y lo moderno, lo local y lo global, lo artesanal y lo tecnológico.

En esa transformación, la segunda mitad del siglo XX es trascendental. Durante ese periodo los tianguis se incrementaron sustancialmente, particularmente en las periferias de las grandes zonas urbanas del país donde se asentaban grandes núcleos de población de escasos recursos. En ello confluyeron varios factores. Por un lado, la incapacidad de las autoridades gubernamentales para ordenar el explosivo crecimiento urbano y, en consecuencia, la ausencia de estrategias para organizar el abasto y distribución de productos básicos, particularmente la incompetencia para construir mercados públicos en esas nuevas áreas habitacionales irregulares y ampliar la cobertura de los mercados existentes o de los que se construían en otras zonas de la ciudad con mayor desarrollo económico (Gayoso, 2012; Ayala y Castillo, 2014). En el desarrollo de los tianguis, sobre todo durante la década de los años 1980 y 1990, también influyó el retiro del Estado de la producción y distribución de productos de primera necesidad para la población de escasos recursos, así como el relativo desinterés para ubicarse en las áreas urbanas habitadas por grupos poblacionales de bajos ingresos, por parte de las grandes cadenas de distribución de alimentos que durante esas mismas dos décadas incrementaron su presencia en el país (Delgado, 1992; Vargas y Rodríguez, 2016).

Junto a esos factores contextuales, el desarrollo de los tianguis también se vio favorecido por las ventajas que supone dicha actividad para sus propios 
actores. Para los clientes, un tianguis representa la oportunidad para proveerse de bienes y servicios de proximidad, así como para la interacción y la agregación social. Para los comerciantes, dada la flexibilidad en las formas de trabajo, la fácil entrada y escasa regulación de la actividad, así como la existencia de redes sociales y familiares para incorporarse a la misma, los tianguis representan una oportunidad para el autoempleo y tener ingresos familiares. Desde una perspectiva sectorial, el desarrollo de los tianguis también significa una solución pragmática para la venta de productos artesanales producidos por los mismos comerciantes o adquiridos directamente de pequeños productores, por un lado y, por el otro lado, para la comercialización de ciertos productos que los distribuidores, particularmente los mayoristas, no pueden vender a través de otros canales comerciales (Delgado, 1992; Bayona, 2011; Gayoso, 2009; 2012; Zamudio, 2012; Espinosa, 2014).

Como objeto de análisis, los tianguis son más referidos en los medios de comunicación que en la bibliografía especializada. En los medios, las referencias suelen ser muy intermitentes y generalmente refieren la informalidad de sus actividades comerciales y ciertos problemas asociados como el no pago de impuestos, la afectación de las zonas donde se establecen, la obstrucción de vialidades, la ausencia de higiene y de garantía sobre los productos que ofrecen, la venta de mercancía de origen ilícito, la competencia desleal al comercio establecido, entre otros. En la bibliografía especializada, el tema de los tianguis suele concentrarse en el análisis de casos específicos de diferentes áreas del país o en ciertas generalidades del comercio tradicional en su conjunto. De acuerdo con Espinosa (2014), en la bibliografía pueden distinguirse dos grandes vertientes. Por una parte, los trabajos de corte antropológico e histórico, focalizados en tianguis ubicados en zonas indígenas apartadas de grandes centros urbanos, y que tratan de resaltar la autenticidad o pasado prehispánico de estos espacios comerciales dado que en su oferta siguen prevaleciendo productos elaborados por la propia comunidad donde se ubican (Fabre y Egea, 2015; Molina y Campos, 2016). Por otra parte, los estudios de carácter socio económico, fundamentados en una visión dualista de formalidad-informalidad del trabajo de los comerciantes y 
El comercio tradicional en la zona metropolitana de Guadalajara, M éxico. El caso de los tianguis

sus actividades comerciales (Flores, 2005; Silva, 2006; Gayoso, 2009; Jaime y Calderón, 2010; Bayona, 2011; Zamudio, 2012; Teja y López, 2013).

En el primer caso, se asume que el carácter informal de los tianguis deriva del propio origen de los mismos, toda vez que la mayoría se instalaron, sin suficiente regulación por parte del Estado, por personas que no tenían un trabajo formal o eran desempleados que buscaban generar empleos familiares (Teja y López, 2013). Bajo ese contexto, los tianguis son propicios para actividades laborales sumamente flexibles en términos del horario de trabajo, la forma de vestir, hablar y comunicarse por parte de los trabajadores, las condiciones de seguridad e higiene en el espacio de trabajo, la planeación y ejecución de las actividades, la calidad y precios de los productos y servicios, el nivel de estudios y formación profesional, la estructura organizacional, el monto de los ingresos, la pertenencia y movilidad del lugar de venta, etcétera. Bajo esa perspectiva, si bien los tianguis y sus comerciantes están registrados y pagan una cuota por "uso del piso" a la autoridad municipal, forman parte de la economía informal en la medida que sus actividades comerciales evaden de una $u$ otra forma, las normas establecidas y previstas por el Estado en la materia como, por ejemplo, el registro de los comercios y el pago de impuestos respectivos ante la Secretaría de Hacienda y Crédito Público, la seguridad social y el reparto de utilidades para los empleados, la información veraz y oportuna del origen, calidad y precios de los productos y servicios ofrecidos, entre otros aspectos (Delgado, 1992; Monnet, 2005; Bayona, 2011; Zamudio, 2012).

En el segundo caso, los tianguis lejos de permanecer en una situación caótica de informalidad, son espacios comerciales itinerantes donde el proceso de trabajo se encuentra enmarcado por relaciones contractuales explicitas e implícitas plenamente funcionales (Gayoso, 2009; 2012; Silva, 2006). De esa manera, la operatividad de los tianguis está asociada con la flexibilidad del Estado para dejar funcionar bajo acuerdos informales, algunas actividades generadoras de ingresos, en un medio social donde actividades similares si están reguladas (Silva, 2006; Cota y Navarro, 2015). Desde esa lógica, el funcionamiento de los tianguis se asocia con la capacidad de sus actores para aprovechar tales huecos 
institucionales y generar prácticas de abasto alternativas en zonas populares donde el comercio formal no tiene mucha presencia o es incipiente. Así, la creación y permanencia de los tianguis deriva de la decisión personal de quienes por su trayectoria laboral y capital humano prefieren vincularse al sector informal por las ventajas que obtienen frente a su vinculación con el sector formal (Silva, 2006). En esta operatividad de los tianguis con reglas más vinculadas a procesos de interacción entre personas que a las establecidas por el Estado, también es determinante la existencia de una estructura de redes sociales donde además de los vecinos, la policía, los transeúntes, los inspectores de salubridad, los administradores municipales, los proveedores, los vendedores ambulantes, los comerciantes de otros formatos comerciales, etcétera, los clientes son actores protagónicos; ellos, mediante sus hábitos de compra y prácticas de consumo, validan y legitiman el ejercicio del trabajo de los comerciantes y la dinámica global de los tianguis (Gayoso, 2009; 2012). Por eso, entonces, desde esta concepción ampliada de los tianguis resulta importante identificar los diversos actores participantes, analizar sus formas de interacción y los mecanismos que ellos movilizan para el intercambio y los resultados derivados, incluyendo las posibles áreas de oportunidad para mejorar de forma individual y colectiva.

El presente trabajo tuvo como objeto de estudio los tianguis de la zona metropolitana de Guadalajara ( $\mathrm{zmG}$ ), que es la segunda metrópoli más grande de México y el principal centro urbano comercial de la región centro occidente de este país. Como tal, no solo constituye un espacio representativo del desarrollo paralelo, y la respectiva imbricación, entre los tianguis y el acelerado proceso de urbanización mencionado arriba, sino también para profundizar en la dinámica de uno de los principales canales del denominado comercio tradicional. Así, el objetivo de este trabajo es analizar la importancia de los tianguis en el abasto y distribución de productos básicos de la zmG, incluyendo las características distintivas de los actores involucrados, así como las prácticas y procesos de intercambio que ellos movilizan y los resultados asociados. 
El comercio tradicional en la zona metropolitana de Guadalajara, M éxico. El caso de los tianguis

\section{Materiales y métodos}

Si se considera como variable distintiva el sitio donde cada uno de los tianguis se establece, independientemente de los días de operación a la semana, en la zona metropolitana de Guadalajara existen 288 tianguis, de los cuales 141 se ubican en el municipio de Guadalajara, 69 en el municipio de Zapopan, 42 en el municipio de San Pedro Tlaquepaque y 36 en el municipio de Tonalá. Tomando como unidad de muestreo los comerciantes de dichos tianguis, se entrevistó a 382 tianguistas (Tabla 1) cuyos datos, según los principios del muestreo aleatorio simple (con un nivel de confianza del $95 \%$ y margen de error del $5 \%$ ), son representativos de los 74.424 comerciantes que constituyen el universo total, de acuerdo con los datos que previamente fueron proporcionados por las autoridades de cada uno de los cuatro Ayuntamientos. Con el objetivo de poder generar información de los tianguis como unidades, por un lado y, por otro lado, para optimizar el desplazamiento y trabajo de 3 equipos de cuatro encuestadores cada uno durante ocho semanas de trabajo de campo, se decidió cubrir la muestra con énfasis en 30 tianguis, mismos que fueron seleccionados de manera aleatoria de un directorio que se integró a partir de los datos obtenidos de las áreas administrativas correspondientes de los municipios en cuestión. En cada tianguis se buscó recoger entre 10 a 30 encuestas según el tamaño de los mismos, tratando de respetar la proporcionalidad de sus giros comerciales, mismos que se contabilizaron y clasificaron previo al levantamiento de las encuestas. Las entrevistas se realizaron directamente en los puestos de venta con el objeto de determinar el perfil del comerciante, los datos del local comercial, el proceso de abastecimiento, la evolución del negocio y la valoración de los servicios generales del tianguis como unidad.

Tabla 1. Número de tianguis y tianguistas en la zona metropolitana de Guadalajara.

\begin{tabular}{|l|r|r|r|r|}
\hline Municipio & $\begin{array}{l}\text { Número de } \\
\text { Tianguis }\end{array}$ & $\begin{array}{l}\text { Número de } \\
\text { Tianguistas }\end{array}$ & $\begin{array}{l}\text { Tianguistas } \\
\text { entrevistados }\end{array}$ & $\begin{array}{l}\text { Clientes } \\
\text { entrevistados }\end{array}$ \\
\hline Guadalajara & 141 & 47000 & 226 & 234 \\
\hline Zapopan & 69 & 17678 & 91 & 89 \\
\hline Tlaquepaque & 42 & 8170 & 39 & 38 \\
\hline
\end{tabular}




\begin{tabular}{|r|r|r|r|r|}
\hline Tonalá & 36 & 1516 & 26 & 26 \\
\hline Total & 288 & 74424 & 382 & 387 \\
\hline
\end{tabular}

Fuente: Elaboración propia.

En lo que corresponde a los clientes, dadas las dificultades para conocer el universo y, en consecuencia, determinar un tamaño de muestra, se optó por implementar la denominada muestra espejo, es decir en cada tianguis se entrevistó a un número de clientes relativamente similar al de los comerciantes encuestados, resultando 387 casos, que fueron seleccionados de manera aleatoria en las diferentes franjas horarias de operación del tianguis en cuestión. Las entrevistas se realizaron directamente en el lugar de compra con objeto de determinar el perfil del cliente, sus hábitos de compra, las características del establecimiento habitual de compra y la valoración de la calidad de los servicios ofrecidos por el tianguis.

En general, el trabajo de campo se realizó del 22 de junio al 14 de agosto de 2016 y los datos se tabularon y analizaron con apoyo del software SPSS Statistics 22 así como del ArcGis 10.3.

Por lo que corresponde a los funcionarios municipales, la información correspondiente deriva de 6 entrevistas a profundidad que se realizaron entre los años 2013 al 2016 a igual número de personas responsables del área de tianguis en los cuatro Ayuntamientos de la zona metropolitana bajo estudio. Además de los datos generales sobre la ubicación, nombre y giros comerciales de los tianguis, los datos derivados de estas entrevistas fueron fundamentales para tener una primera aproximación sobre la estructura y operación general de dichos espacios comerciales.

\section{Resultados y discusión}

\section{Generalidades de los tianguis de la zona metropolitana de Guadalajara}

Como se mencionó anteriormente, si se toma como referencia el lugar de la vía pública donde operan, en la zmG existen 288 tianguis, de los cuales 256 (89 \%) solo operan un día a la semana, 22 trabajan 2 días a la semana y 10 funcionan de 3 a 7 días a la semana (Tabla 2). 
El comercio tradicional en la zona metropolitana de Guadalajara, M éxico. El caso de los tianguis

Tabla 2. Cantidad de tianguis según el número de días que operan por semana

\begin{tabular}{|c|c|c|c|c|c|c|c|c|}
\hline \multirow{2}{*}{ Municipio } & \multirow{2}{*}{\begin{tabular}{|l|}
$\begin{array}{l}\text { Total de } \\
\text { tianguis }\end{array}$ \\
\end{tabular}} & \multicolumn{7}{|c|}{ Número de días que operan a la semana } \\
\hline & & 1 & 2 & 3 & 4 & 5 & 6 & 7 \\
\hline Guadalajara & 141 & 128 & 11 & 1 & 0 & 0 & 0 & 1 \\
\hline Zapopan & 69 & 66 & 2 & 1 & 0 & 0 & 0 & 0 \\
\hline Tlaquepaque & 42 & 39 & 2 & 1 & 0 & 0 & 0 & 0 \\
\hline Tonalá & 36 & 23 & 7 & 4 & 1 & 1 & 0 & 0 \\
\hline Total & 288 & 256 & 22 & 7 & 1 & 1 & 0 & 1 \\
\hline
\end{tabular}

Fuente: Elaboración propia.

Si se toma en cuenta todos los días que funcionan, diariamente están activos entre 42 a 56 tianguis, siendo los domingos y los viernes, los días de mayor y menor número, respectivamente $\mathrm{y}$, en consecuencia, a lo largo de la semana pueden contabilizarse 334 jornadas de venta por parte de los tianguis ubicados en la zona metropolitana de Guadalajara. Vale destacar que con excepción del tianguis Felipe Ángeles, que opera los siete días de la semana, en el resto de los tianguis que se establecen en el mismo sitio durante dos o más días a la semana, una parte de sus comerciantes varía entre un día y otro, generalmente porque uno de esos días de operación suele incrementarse la demanda de sus productos y, por tanto, se incorporan comerciantes de otros tianguis $y$, en menor medida, vecinos del lugar donde se ubican.

Del total de tianguis, una quinta parte se integra por 6 a 99 comerciantes, el $65 \%$ por 100 a 299 comerciantes, el $15 \%$ por 300 a 999 comerciantes y el $2 \%$ por 1000 a cerca de 10000 comerciantes. En consecuencia, la extensión de cada tianguis puede variar desde unos 30 metros lineales hasta cerca de nueve kilómetros, como es el caso del tianguis "El Baratillo", que es el de mayor número de comerciantes y constituye uno de los más grandes del país (Espinosa, 2014).

Si bien el tamaño de los tianguis puede guardar una relación relativamente proporcional con la diversidad de giros y servicios que ofrecen, existen excepciones que se especializan en la venta de uno o muy pocos productos, como es el caso del tianguis de la "Calle 66" orientado a la venta de ropa y el tianguis de 
"Tonalá" especializado en la venta de artesanías; ambos reconocidos entre los más importantes de América Latina, en su ámbito de especialización.

En la Figura 1, se aprecia que si bien los tianguis están distribuidos por todo el territorio de la ZMG la mayoría se concentran en la periferia de cada municipio y particularmente en la franja fronteriza entre Guadalajara y Tlaquepaque y Guadalajara y Tonalá, aspecto que se mantiene desde mediados de la década de los años 1990, cuando las autoridades municipales decidieron no otorgar más permisos para la apertura de nuevos tianguis (Flores, 2005).

Figura 1. Distribución de los tianguis en la zmG

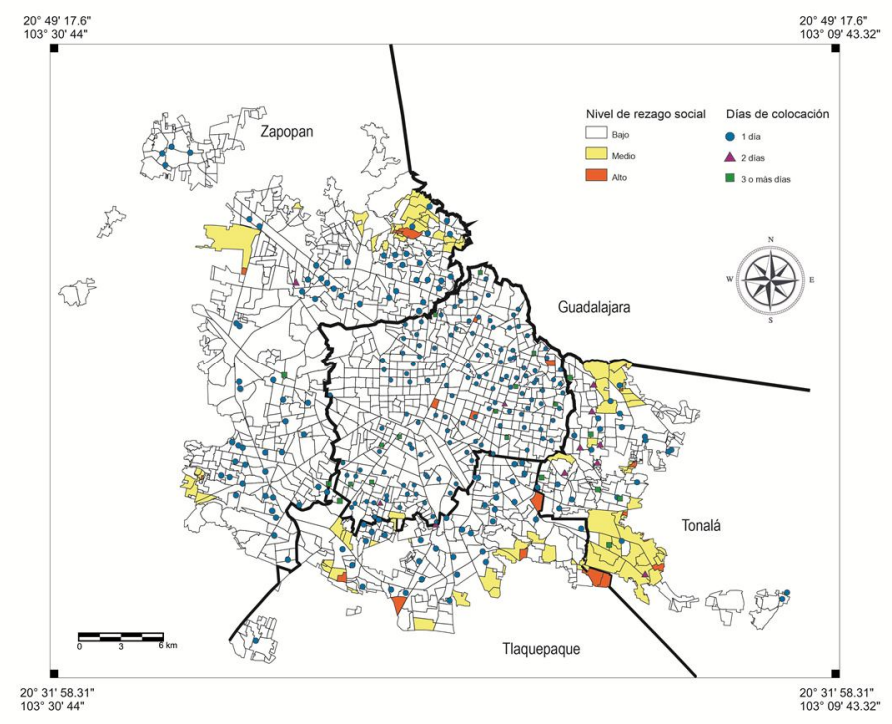

Fuente: Elaboración propia.

\section{Los tianguistas y sus negocios}

Perfil de los comerciantes y de los puestos de venta

Los 30 tianguis bajo estudio albergan 5358 puestos de venta de productos de 33 giros diferentes, destacando tanto por su cantidad como por su presencia prácticamente en todos los tianguis, los de ropa nueva y usada, frutas y verduras, calzado, cuidado e higiene personal, comida preparada y artículos para el hogar (Tabla 3). El $96 \%$ de los locales son atendidos directamente por sus propietarios 0 algún familiar directo y el $4 \%$ restante por un arrendatario; estos últimos casos se concentran en los comercios de ropa, frutas y verduras y artículos para el hogar, 
El comercio tradicional en la zona metropolitana de Guadalajara, M éxico. El caso de los tianguis

principalmente $\mathrm{y}$, en menor medida, en los de calzado, higiene personal y videojuegos.

Tabla 3. Número de locales según su principal giro comercial

\begin{tabular}{|l|r|r|l|r|r|}
\hline Tipo de giro & Número & \multicolumn{1}{l|}{$\%$} & Tipo de giro & Número & $\%$ \\
\hline $\begin{array}{l}\text { Ropa (nueva y de } \\
\text { segunda) }\end{array}$ & 2035 & 37.98 & Pollo y/o huevo & 44 & 0.82 \\
\hline Frutas y verduras & 554 & 10.34 & Telefonía y servicios & 44 & 0.82 \\
\hline Calzado & 311 & 5.80 & Pescados y mariscos & 38 & 0.71 \\
\hline $\begin{array}{l}\text { Cuidado e higiene } \\
\text { personal }\end{array}$ & 308 & 5.75 & Revistas & 34 & 0.63 \\
\hline Comida preparada & 295 & 5.51 & Tostadas y frituras & 33 & 0.62 \\
\hline Artículos para el hogar & 282 & 5.26 & $\begin{array}{l}\text { Artículos de limpieza } \\
\text { domestica }\end{array}$ & 31 & 0.58 \\
\hline Joyería & 234 & 4.37 & Artículos naturistas & 25 & 0.47 \\
\hline Electrónica y videojuegos & 227 & 4.24 & Dulcería & 23 & 0.43 \\
\hline Artículos usados & 150 & 2.80 & Mueblería & 21 & 0.39 \\
\hline Productos de plásticos & 139 & 2.59 & Papelería & 17 & 0.32 \\
\hline Flores & 85 & 1.59 & Carnes rojas & 14 & 0.26 \\
\hline $\begin{array}{l}\text { Jugos y bebidas } \\
\text { preparadas }\end{array}$ & 82 & 1.53 & Panadería & 14 & 0.26 \\
\hline Semillas y cereales & 71 & 1.33 & Encurtidos & 14 & 0.26 \\
\hline Abarrotes & 58 & 1.08 & Herramientas & 8 & 0.15 \\
\hline $\begin{array}{l}\text { Productos lácteos y } \\
\text { derivados }\end{array}$ & 57 & 1.06 & Cerrajería & 7 & 0.13 \\
\hline $\begin{array}{l}\text { Mascotas y animales de } \\
\text { corral }\end{array}$ & 51 & 0.95 & Tortillería & 3 & 0.06 \\
\hline Mercería & 49 & 0.91 & Total & 5358 & 100 \\
\hline
\end{tabular}

Fuente: Elaboración propia.

La edad promedio de los comerciantes es de 44 años, destacando el grupo etario de 30 a 49 años (55\%), las mujeres (55\%), los casados (75\%) y quienes tienen estudios de secundaria (40\%) (Tabla 4). El $73 \%$ de los entrevistados manifestó tener más de 5 años como comerciante en los tianguis, destacando que mientras en este grupo prácticamente hay un porcentaje similar de mujeres y de hombres, en el grupo de comerciantes con menos de 5 años de antigüedad es mayor el porcentaje de mujeres (65\%) que el de los hombres (35\%): dado que el $82 \%$ de estas mujeres son casadas (82\%) y cerca del $70 \%$ venden ropa, podemos subrayar la importancia de sumar más miembros de la familia a las actividades productivas para favorecer el ingreso familiar, aprovechando las facilidades de entrada y salida en el ejercicio de la principal actividad comercial 
que ocurre en los tianguis. En este sentido resalta que el $67 \%$ de los tianguistas manifestó dedicarse a esta actividad comercial por la necesidad de contar con un empleo remunerado, mientras que $19 \%$ por considerarlo un buen negocio, $11 \%$ por tradición familiar y $3 \%$ por la cercanía a su domicilio. Tales expresiones sobre la incorporación a estas actividades por las dificultades de empleo en el sector formal, junto a la notable participación de las mujeres y la movilización de las redes familiares en un contexto de acuerdos y reglas de operación informales, incluso ante las autoridades municipales, puede ser una muestra de la adaptación de ciertos sectores de la población a contextos socioeconómicos en situaciones de precariedad (Food and Agriculture Organization, 2003).

Tabla 4. Perfil de los comerciantes de los tianguis de la ZMG

\begin{tabular}{|c|c|c|c|}
\hline Variable & Grupo/Indicador & $\%$ & $\%$ acumulado \\
\hline \multirow{4}{*}{ Edad } & 15 a 29 años & 11 & 11 \\
\hline & 30 a 49 años & 55 & 66 \\
\hline & 50 a 64 años & 26 & 92 \\
\hline & 65 y más años & 8 & 100 \\
\hline \multirow[b]{2}{*}{ Género } & Femenino & 55 & 55 \\
\hline & Masculino & 45 & 100 \\
\hline & & & \\
\hline \multirow{3}{*}{ Estado civil } & Casado (a) & 75 & 75 \\
\hline & Soltero (a) & 17 & 92 \\
\hline & Viudo (a) o divorciado (a) & 8 & 100 \\
\hline \multirow{5}{*}{ Escolaridad } & Sin estudios & 6 & 6 \\
\hline & Primaria & 22 & 28 \\
\hline & Secundaria & 40 & 68 \\
\hline & Bachillerato & 27 & 95 \\
\hline & Licenciatura & 5 & 100 \\
\hline
\end{tabular}

Fuente: Elaboración propia.

Dentro de un mismo tianguis, el $95 \%$ de los comerciantes solo tiene un puesto y el $5 \%$ restante tienen dos locales, a excepción de un caso que manifestó tener tres puestos comerciales en el mismo tianguis. No obstante, solamente el 19 $\%$ de ellos vende únicamente en un tianguis, $16 \%$ lo hace en dos, $22 \%$ en tres, $21 \%$ en cuatro y $22 \%$ en cinco o más tianguis a la semana. De acuerdo con Teja y López (2013), con esta estrategia los comerciantes incrementan sus nichos de mercado y el alcance de sus bienes entre una mayor población consumidora. 
El comercio tradicional en la zona metropolitana de Guadalajara, M éxico. El caso de los tianguis

\section{Operatividad de los puestos comerciales}

Alrededor de una cuarta parte de los comerciantes entrevistados comienza a vender de 6:00 a 8:00 de la mañana y el $75 \%$ restante inicia de 8:00 a 9:00 horas. El $22 \%$ deja de vender antes de las 15:00 h, el $75 \%$ de las 15:00 a las 16:00 h y el $3 \%$ restante de 16:00 a 18:00 h. En lo que corresponde a la superficie, el $56 \%$ tiene menos de $4 \mathrm{~m}^{2}$, el $40 \%$ oscila entre $4 \mathrm{~m}^{2}$ y $12 \mathrm{~m}^{2}$ y el $6 \%$ restante tiene de $13 \mathrm{~m}^{2}$ a $30 \mathrm{~m}^{2}$; entre estos de mayor tamaño destacan los puestos que venden artículos para el hogar, ropa, comida preparada y frutas y verduras. Esta diversidad en la oferta, pero también en el tamaño, horario de apertura y cierre $y$, sobre todo, en los medios y estrategias de marketing que cada comerciante implementa, si bien muestra la improvisación imperante también contribuye para instaurar relaciones cercanas entre los concurrentes $y$, en consecuencia, en un espacio urbano para influenciarse mutuamente (Flores, 2005; Espinosa, 2014).

En promedio, en cada puesto trabajan 1.8 personas, sobresaliendo que el $98 \%$ tiene entre 1 a 4 empleados, que en ningún caso hay más de siete personas laborando y que en el $90 \%$ de los puestos únicamente laboran su propietario o algún miembro de su familia. En aquellos casos donde se cuenta con algún empleado ( $10 \%$ de los puestos), solo el $23 \%$ asegura otorgarles aguinaldo, el 8 $\%$ vacaciones y el $3 \%$ seguro médico. Ello, junto a la ausencia de prestaciones para los miembros de la familia que participan en el negocio, subraya el carácter informal de estas actividades laborales.

En general, el mobiliario utilizado para exposición de los productos es precario y el equipo más común son las basculas mecánicas para pesaje de frutas y verduras, pollo y huevo, así como equipo de refrigeración, con cierto grado de deterioro, particularmente en una parte de los puestos especializados en la venta de productos lácteos y derivados ( $86 \%$ ) y de comida preparada (40\%).

Si bien la autoridad municipal es responsable de autorizar la apertura de los tianguis y los puestos comerciales que estos albergan, alrededor del $35 \%$ de los comerciantes entrevistados adquirió su puesto de venta mediante traspaso, 
principalmente, o arrendamiento, en menor medida, de otros tianguistas registrados directamente ante el Ayuntamiento. De esa manera, estos comerciantes evitan un trámite administrativo y, sobre todo, el pago de cesión de derechos ante la autoridad municipal.

En todos los tianguis, los comerciantes expresaron la existencia de un reglamento municipal, particularmente sobre el horario y algunas restricciones sobre el uso de su espacio de venta. No obstante, $14 \%$ de los comerciantes, en promedio, de dos terceras partes de los tianguis manifestaron desconocer la existencia de algún reglamento específico para la operación de los tianguis.

En términos organizacionales, 6 tianguistas declararon la existencia de una asociación de comerciantes en el tianguis donde laboraban, sin embargo, al ser cuestionados sobre su participación y periodicidad de las reuniones de trabajo, apenas uno de ellos aseguró participar en la misma. Así, podríamos considerar que alude a una agrupación ajena al mismo tianguis o bien que la referida no incluye a todos los comerciantes del mismo.

Cada día de operación, los comerciantes deben pagar al representante de la autoridad municipal una "cuota de uso de piso", directamente en su puesto de venta. Si bien la Ley de Ingresos de cada Ayuntamiento define el monto de la misma $y$, en principio, es similar para todos los tianguis de Tonalá y es diferenciada, por zona de ubicación, en el caso de Guadalajara, Zapopan y Tlaquepaque, nuestros datos refieren que existe cierta diferenciación al interior de los tianguis, particularmente dependiendo de la ubicación, el giro comercial del puesto $\mathrm{y}$, probablemente, las habilidades de "regateo" del comerciante en cuestión. Así, aunque el promedio de dicha cuota es de $\$ 6.00$ por $\mathrm{m}^{2}$, alrededor del $3 \%$ de los entrevistados aseguró no hacer ningún tipo de pago, y entre los que si lo hacen las variaciones van desde $\$ 1.00$, en todos los municipios, hasta $\$ 8.00$ en Tlaquepaque, $\$ 10.00$ en Tonalá, $\$ 15.00$ en Zapopan y $\$ 25.00$ en Guadalajara.

Además de la cuota por uso de piso, alrededor del $20 \%$ de los comerciantes entrevistados manifestaron hacer una aportación extraordinaria, particularmente para la recolección de basura. Así, las labores de limpieza son 
El comercio tradicional en la zona metropolitana de Guadalajara, M éxico. El caso de los tianguis

cubiertas en gran parte con recursos del propio Ayuntamiento sino es que también llega ser una externalidad negativa para los propios vecinos.

Esta laxitud en la aplicación de la norma por parte de la autoridad municipal, evidencia lo apuntado por Gómez (2007) y Bayona (2011) respecto del alto contenido político que caracteriza al comercio en vía pública y, en consecuencia, las dificultades para generar alternativas de política pública y regular este tipo de actividades comerciales.

\section{Abasto y traslado de los productos al punto de venta}

El $39 \%$ de los comerciantes se abastecen directamente de la fábrica, empresa de distribución o productor directo de los bienes que venden. El resto de la proveeduría se realiza en el Mercado de Abastos de Guadalajara y de otros comerciantes minoristas de los mercados municipales, tianguis e incluso de abarrotes. A nivel de giro comercial, se incrementa la participación de las empresas o fabricantes directos de ropa importada, calzado, cuidado e higiene personal, ropa nueva y artículos para el hogar, por un lado y, por otro lado, del mercado de abastos, mercados municipales, otros comerciantes minoristas y tianguis, para el caso de frutas y verduras, comida preparada y ropa de segunda.

Para trasladar los productos al punto de venta, el $68 \%$ de los comerciantes utiliza vehículo particular, el $14 \%$ lo hace en transporte público, el $12 \%$ lo hace "a pie", ya sea cargando o con apoyo de una carretilla o diablito de carga, y el $6 \%$ restante mediante algún vehículo alquilado o prestado.

La periodicidad del abastecimiento de sus productos está determinada en gran medida por el número de días de venta a la semana, así como por el tipo de giro. De tal forma, por ejemplo, mientras que todos los comerciantes de productos muy perecederos, como alimentos preparados y frutas y verduras, se surten al menos una vez a la semana, con mayor grado de recurrencia entre los que venden en varios tianguis, más del $50 \%$ de los comerciantes de productos no perecederos, como calzado y ropa nueva e importada, se surten cada 15 a 30 días o más. Esta situación no solo reafirma el carácter minorista de la actividad, sino que también es una expresión de la baja durabilidad de los productos que se 
ofertan en estos espacios comerciales y, particularmente, de los vínculos que se establecen entre el comercio formal y mayorista y el minorista informal, así como de las singularidades de los tianguis para cubrir el abasto doméstico tanto de productos básicos como no tan necesarios (Bayona, 2011).

Evolución de las ventas y áreas de mejora

En promedio, cada puesto atiende a 28 clientes por día, sobresaliendo que en $84 \%$ de los puestos comerciales dicho número es inferior a 50 y que solamente el $3 \%$ supera los 90 , pero ninguno sobrepasa los 200 clientes por día de venta. Mientras que en los giros con menor afluencia de consumidores sobresalen los que venden ropa, calzado, artículos para el hogar, papelería, y mascotas, en los de mayor número destacan los de comida preparada y de frutas y verduras. De acuerdo con los propios comerciantes, el $49 \%$ de los clientes gasta menos de 50 pesos en cada puesto, el $36 \%$ entre $\$ 50.00$ y $\$ 100.00$, el 12 $\%$ entre $\$ 101.00$ y $\$ 200.00$, y solamente el $3 \%$ entre $\$ 201.00$ y $\$ 1000.00$. En consecuencia, $73 \%$ de los comerciantes aseguran no vender más de \$1000.00 por día, $25 \%$ venden entre $\$ 1000$ y $\$ 4000$ y solo el $2 \%$ un monto que oscila entre $\$ 4000.00$ y \$ 10000.00 en cada día de operaciones. En este último grupo solamente se encuentran quienes venden ropa nueva, usada e importada, así como comida preparada. Bajo estas condiciones, podemos considerar que ejercer la actividad comercial en los tianguis es una opción donde más que ganar dinero, se pueden obtener recursos para la subsistencia, jornada a jornada (Bayona, 2011).

De acuerdo con los datos disponibles, entre las fortalezas que sostienen sus negocios, $81 \%$ de los comerciantes destaca el trato amable y los precios bajos de sus productos. El $19 \%$ restante considera que sus clientes acuden al tianguis ya sea porque la mercancía está a la vista y alcance de su mano o porque dan degustaciones, pilones e, incluso, rebajas de precios a lo largo de una jornada de venta. Esto, de alguna manera, reafirma que la calidad de los productos no constituye una de las fortalezas distintivas de estos establecimientos comerciales. 
El comercio tradicional en la zona metropolitana de Guadalajara, M éxico. El caso de los tianguis

Entre las condiciones inherentes al tianguis en su conjunto, la mayoría de los comerciantes tienen buena percepción sobre su funcionamiento, sobresaliendo entre los rubros con mayor número de insatisfechos o muy insatisfechos la actuación por parte de la autoridad municipal y la inseguridad.

En lo que corresponde a sus interacciones con los demás comerciantes del mismo tianguis, prácticamente todos subrayan el buen trato y cooperación entre sí, y entre los que tendrían algún cuestionamiento para quienes tienen un puesto vecino es, principalmente, sobre los gritos para promover sus productos, la limpieza de sus espacios, la invasión de espacios comunes, la variación constante de precios y la música en alto volumen. No obstante, 71 y $8 \%$, respectivamente, consideran como su principal competencia a los otros comerciantes y vendedores ambulantes del mismo tianguis; otro $13 \%$ considera como su principal competidor a los supermercados y el $8 \%$ restante a los abarrotes, mercados municipales y tiendas de conveniencia cercanas al tianguis. Siguiendo a Sanclemente (2014), podemos considerar que esta percepción sobre la mayor competencia con otros formatos del comercio tradicional, a pesar de la proliferación de establecimientos de las grandes cadenas de distribución minorista, bien puede ser una expresión de la rivalidad que ellos ven con quienes les disputan el mismo segmento de consumidores.

Bajo el contexto anterior, llama la atención que cerca del $10 \%$ de los entrevistados consideraba que no era necesario algún cambio para mejorar las ventas en el tianguis donde venden. $30 \%$ de los que se manifestaron por algún cambio, subrayaron que el financiamiento y la mejora de los servicios públicos serían la mejor opción para optimizar su actividad comercial, seguida de la mejora en la calidad, precio y variedad de productos (25\%), seguridad y limpieza (19\%), publicidad y técnicas de venta (13\%), cambios en los días de venta del tianguis $(11 \%)$ y otros $(2 \%)$.

Para resaltar la importancia que tienen los tianguis en la sociedad y, en consecuencia, justificar la permanencia de estos espacios comerciales, $60 \%$ enfatiza su rol en la generación de ingresos y empleos familiares, $21 \%$ destaca su 
función en el abasto de productos básicos para los hogares y $20 \%$ como espacios tradicionales que propician la convivencia social.

\section{Los clientes de los tianguis}

Perfil general de los clientes de los tianguis

La edad promedio de los clientes de los tianguis es de 42 años, sobresaliendo el grupo etario de 30 a 49 años (46\%) y las mujeres (73\%). Los clientes pertenecen a hogares con 4 miembros en promedio, el $22 \%$ no tiene estudios mayores al nivel de primaria y solamente el $20 \%$ cursó estudios universitarios. Destaca que cerca el $13 \%$ de los entrevistados manifestó no contar con un empleo al momento de la entrevista y que, de acuerdo con los ingresos familiares, el $61 \%$ de todos los clientes entrevistados son pobres, el $38 \%$ son clase media y el $1 \%$ restante son ricos, lo cual ratifica el rol destacado de estos espacios comerciales en la función de abasto de las clases populares.

Hábitos de compra de los consumidores de los tianguis

Alrededor del $30 \%$ de los entrevistados han sido clientes del tianguis donde fueron encuestados por más de 10 años, incrementándose tal porcentaje hasta el 50 \% en la población de más de 50 años de edad. Resulta obvio que la fidelidad de la población joven sea menos añeja por su misma restricción de la edad, no obstante pudiera suponerse que el vínculo de los entrevistados con los tianguis no es más longevo, sobre todo entre los grupos de mayor edad, tanto porque los tianguis, e incluso la colonia donde se ubican, son relativamente recientes, comparados, por ejemplo, con los mercados municipales, como por el constante cambio de domicilio de una parte de estos consumidores, toda vez que carecen de vivienda propia.

Mientras dos terceras partes de los clientes acuden al tianguis donde fueron entrevistados, el día o días de la semana en que éste opera, $22 \%$ lo hace 1 o 2 veces por mes y el $12 \%$ restante de manera ocasional. Cuestionados sobre su grado de fidelidad con un mismo comerciante dentro del tianguis, una tercera parte de los entrevistados manifestó comprar habitualmente en el mismo puesto, sobre 
El comercio tradicional en la zona metropolitana de Guadalajara, M éxico. El caso de los tianguis

todo para adquirir frutas y verduras o alimentos preparados. Para hacer sus compras, la mitad de los consumidores acuden solos, $44 \%$ acompañados de un familiar, sobre todo de su pareja o hijos, y $6 \%$ con algún vecino o amigo.

En cuanto a su lugar de residencia, $98 \%$ de los clientes entrevistados viven en la zona metropolitana de Guadalajara y el $2 \%$ fuera de la misma. De los que viven en esta metrópoli, $94 \%$ viven en la misma colonia o una colonia vecina al lugar donde se ubica el tianguis. Dada tal cercanía y, en menor medida, las dificultades para encontrar estacionamiento o por no disponer de otra opción, el 71 $\%$ se traslada al tianguis caminando, $18 \%$ en vehículo particular, $9 \%$ en transporte público y $2 \%$ en bicicleta o motocicleta.

Para realizar sus compras, $33 \%$ prefiere hacerlo antes de las 10 de la mañana, la mitad de las 10 a 12 horas, el $14 \%$ de 12 h a 14 h, y el $3 \%$ restante entre las 14 y $16 \mathrm{~h}$. El $98 \%$ adquiere productos para el consumo familiar y $2 \%$ para comercializarlos, particularmente ropa, comida preparada y frutas y verduras. En promedio, durante una jornada de compra cada cliente gasta $\$ 230.00$ y si bien el $6 \%$ suele comprar más de $\$ 500.00$, ninguno supera los $\$ 1500.00$ (Figura 2 ).

Figura 2. Monto del gasto por ocasión de compra en los tianguis

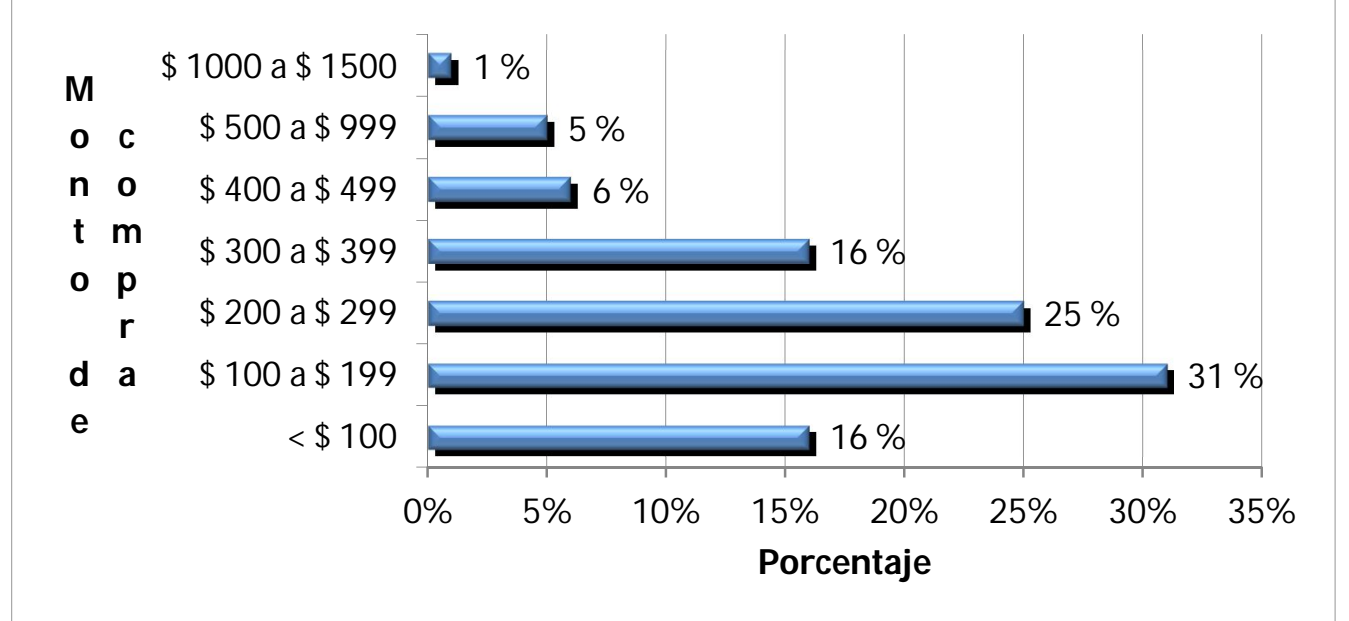

Fuente: Elaboración propia.

Tomando en consideración el principal producto que los consumidores compran en el tianguis, sobresalen las frutas y verduras, la ropa nueva, la comida preparada, la ropa de segunda y los lácteos y derivados. Por supuesto, en los tianguis también se intercambian otros productos no básicos y bienes usados, 
como herramientas, juguetes, aparatos electrónicos, etcétera, lo que expresa no solamente la penuria de los compradores sino también lo que Licona (2014) considera un sistema de intercambio socializante desarrollado por sectores pobres.

Motivos de compra y nivel de satisfacción con la calidad de los tianguis

Cuestionados sobre las razones por las cuales deciden comprar en los tianguis, $40 \%$ de los clientes refirieron la cercanía a su domicilio, $38 \%$ los buenos precios, $11 \%$ la calidad de los productos, $3 \%$ la variedad de productos y el $8 \%$ restante diversos factores como la costumbre, las ofertas, la comodidad y el trato amable.

Frente a las percepciones de los comerciantes sobre las razones por las cuales consideran que sus clientes los eligen, vale destacar el bajo porcentaje de consumidores que manifiestan como prioridad el trato amable por parte de los tianguistas, la relativa coincidencia sobre la importancia de los precios, así como la mayor importancia que los clientes proporcionan a la calidad y variedad de los productos. La relevancia de la cercanía solo ratifica la ventaja de proximidad que tienen estos espacios comerciales. El significado como lugares públicos para la interacción y agregación social queda manifiesto en tanto que cerca de la mitad de los entrevistados considera que su visita al tianguis, además del abasto, también ofrece una oportunidad para distraerse, compartir tiempos con conocidos, hacer ejercicio o un simple gusto para salir de las actividades rutinarias.

Entre los establecimientos comerciales donde los consumidores de los tianguis también compran los productos de su canasta básica, destacan entre los más recurridos las grandes cadenas comerciales y los abarrotes, principalmente y, en menor medida, los mercados municipales y las tiendas de conveniencia. En general, los criterios de elección de esos establecimientos se relacionan con la cercanía, precios bajos y calidad y variedad de los productos. Esta escasa diferenciación respecto de los establecimientos del comercio tradicional, por lo menos en la comodidad de compra, posiblemente influye el hecho que el formato de tienda que las cadenas comerciales han venido implementando en las zonas 
El comercio tradicional en la zona metropolitana de Guadalajara, M éxico. El caso de los tianguis

populares no guarda marcadas diferencias con los establecimientos tradicionales, como, por ejemplo, los abarrotes, en lo que corresponde a la accesibilidad, estacionamiento y condiciones del inmueble.

Sobra decir que en esta diversificación de los establecimientos de abasto de la canasta básica también influyen las propias condiciones de la oferta comercial y los servicios que los clientes reciben en los tianguis. Al respecto, cuestionados sobre sus planes para seguir comprando en los tianguis en los próximos tres años, a partir del trato que reciben por parte de los comerciantes, las características de los productos que se ofertan y, sobre todo, las condiciones de los tianguis en su conjunto, $90 \%$ de los entrevistados manifestaron una marcada intención de continuar proveyéndose en estos espacios comerciales. Como áreas de mejora estos clientes destacan particularmente la limpieza, la imagen de los puestos, diversificar la oferta de productos, la seguridad y los servicios públicos. Para el $10 \%$ que expresó su desanimo para seguir adquiriendo productos en los tianguis, además de los factores anteriores mencionaron la calidad de los productos, así como las ofertas y promociones. De acuerdo con la propuesta de Bocanegra (2016) esta percepción, además de las propias deficiencias que los clientes visualizan en la operatividad de los tianguis, bien puede estar enmarcada por las estrategias que las grandes cadenas de distribución minorista han utilizado para conquistar la preferencia del consumidor mexicano.

\section{Conclusiones}

La emergencia y desarrollo de los tianguis está vinculada a factores estructurales donde resalta el escaso interés e incluso la incapacidad del Estado para organizar el abasto y distribución de alimentos, particularmente en las áreas urbanas de alta densidad poblacional.

Aprovechando los huecos institucionales $y$, por tanto la relativa facilidad para emprender esta actividad comercial, los tianguis han resultado un espacio propicio para el autoempleo y la obtención de ingresos familiares mediante formas de trabajo sumamente flexibles en términos de horario, nivel educativo y formación, inversión, equipo y mobiliario y estructura organizacional. 
Bajo esas condiciones representan una de las mejores expresiones del comercio tradicional cuya permanencia está menos vinculada con la calidad de los bienes y servicios ofertados que con las ventajas de proximidad con una demanda orientada fundamentalmente por la cercanía y, con ello, una percepción de ser espacios públicos para convivir con otros y distraerse de las actividades cotidianas. Bajo ese marco, paradójicamente, los tianguis dan pie a un complejo conjunto de prácticas y procesos de intercambio donde los clientes, e incluso los oferentes, aceptan falta de limpieza, inseguridad, mal arreglo de los puestos comerciales, baja durabilidad de los productos, mala relación calidad precio de los productos y afectación de las vialidades públicas.

Frente a la competencia que representan las grandes cadenas comerciales, particularmente con la apertura de puntos de venta en pequeños formatos, sobra decir que esa ventaja de proximidad puede irse mermando y, en consecuencia, su devenir podría ser más promisorio en la medida que estas prácticas y procesos de intercambio favorecieran aspectos que potenciarán las amenidades asociadas con el buen trato de los comerciantes del sector tradicional, la mejora en la calidad de los servicios y, particularmente, con el origen y seguridad de los alimentos que allí se consumen y se adquieren para preparar en casa. En ello, vale tener presente las bondades de la responsabilidad social entre oferentes y demandantes, pero también es importante recordar la actuación de las autoridades locales, en tanto los tianguis se ubican en espacios públicos y ofrecen un servicio a la comunidad, para estimular la creación y consolidación de reglas y normas que regulen las interacciones con otros actores, particularmente los comerciantes de otro tipo de establecimientos cercanos y los vecinos de los tianguis.

\section{Referencias Bibliográficas}

Ayala, Suhey y Castillo, Víctor (2014). La distribución de alimentos y bebidas en México: una perspectiva desde el comercio tradicional. Espacio Abierto. Cuaderno Venezolano de Sociología. Volumen 23, No. 4. Venezuela (Pp. 661-681).

Bayona, Eugenia (2011). Comercio y consumo en la periferia de la zona metropolitana de Guadalajara. Revista de Antropología Experimental. No. 11. España (Pp. 283-298). 
El comercio tradicional en la zona metropolitana de Guadalajara, M éxico. El caso de los tianguis

Bocanegra, Carmen (2016). El papel de las empresas trasnacionales en el comercio minorista. La walmartización en México. Equilibrio económico. Revista de Economía, Política y Sociedad. Volumen 12, No. 1. México (Pp. 29-52).

Cota, Rosario y Navarro, Alberto (2015). Análisis del mercado laboral y el empleo informal mexicano. Papeles de población. Volumen 21, No. 85. México (Pp. 211-249).

Delgado, Ranulfo (1992). La distribución de bienes de consumo generalizado en la ciudad de México. Del tianguis al tianguis. Revista de Administración Pública. No. 83. México (Pp. 167-174).

Espinosa, Horacio (2014). Piel de Calle. Una deriva en el tianguis Baratillo, Revista de Estudios Urbanos y Ciencias Sociales. Volumen 4. No. 2. España (Pp. 25-47).

Fabre, Danú y Egea, Carmen (2015). Los espacios de intercambio. Los tianguis de Páztcuaro (Michoacán, México), entre la tradición y las estrategias de supervivencia. Documents d'Anàlisi Geogràfica. Volumen 61. No. 2. España (pp. 265-287).

Food and Agriculture Organization (2003). El sector informal alimentario. Roma. FAO. Italia.

Flores, Manuel (2005). Tianguis, mercados y tenderetes en Jalisco. En Luis Antonio González (compilador), Encuentros sociales y diversiones (pp. 87107). Secretaría de Cultura del Estado de Jalisco. México.

García, Rocio; Rappo, Susana y Temple, Ludovic (2016). Innovaciones socioambientales en el sistema agroalimentario de México: los mercados locales alternativos (tianguis). Agroalimentaria. Volumen 22. No. 43. Venezuela (Pp. 103-117).

Gayoso, José Luis (2009). Los tianguistas de la Ciudad de México: de informales a trabajadores atípicos. Iztapalapa. No. 66. México (Pp. 53-67).

Gayoso, José Luis (2012). Trabajo, identidad y acción colectiva en el trabajo no clásico: los vendedores de tianguis en el D.F. Tesis de doctorado en Estudios Sociales, México, Universidad Autónoma Metropolitana. [En línea]. Disponible en:

http://www.izt.uam.mx/sotraem/NovedadesEditoriales/TesisDoctoradoGayosso. pdf. Consulta: 10/9/2016.

Gómez, Norma Angélica (2007). Redes sociales y comercio en vía pública en la ciudad de México. El Cotidiano. Volumen 22. No. 143. México (Pp. 41-47).

Jaime, Nora y Calderón, Salvador (2010). Economía Informal: el caso del ambulantaje ¿existe? México, Fundación Rafael Preciado Hernández A.C. Documento de trabajo No. 370. [En línea]. Disponible en: http://frph.org.mx/bdigital/verpdf.php?cve=370. Consulta: 5/9/2016.

Licona, Ernesto (2014). Un sistema de intercambio híbrido: el mercado/ tianguis La Purísima, Tehuacán-Puebla, México. Antípoda. No. 18. Colombia (Pp. 137163).

Molina, Nancy Gabriela y Campos, Gisela Virginia (2016). Historia y situación actual de los mercados semanales en los valles centrales de Oaxaca. Revista

Mexicana de Agroecosistemas. Volumen 3. No. 2. México (Pp. 272-290).

Monnet, Jérôme (2005). Conceptualización del ambulantaje, de los vendedores a 
los clientes: un acercamiento universidad a la metrópoli posfordista. En J. Monnet y J. Bonnafe (Ed.), Memoria del Seminario: El ambulantaje en la Ciudad de México. Investigaciones recientes (pp. 13-26). Centro Francés de Estudios Mexicanos y Centroamericanos / Programa Universitario de Estudios de la Ciudad de la Universidad Nacional Autónoma de México. México.

Sanclemente, Juan Carlos (2014). Los pequeños distribuidores minoristas y su aporte en la realización de Negocios Inclusivos en Colombia. Revue Interventions économiques. No. 50. Canadá (Pp. 1-26).

Silva, Diana Alejandra (2006). Espacio urbano y comercio en vía pública. Reglas, redes y uso del espacio público en la ciudad de México. Tesis de Maestría en Ciencias Sociales. México, FLACSO. [En línea]. Disponible en: http://conocimientoabierto.flacso.edu.mx/medios/tesis/silva da.pdf. Consulta: 23/10/2016.

Teja, Rebeca y López, Nidia (2013). Comercio informal: un estudio en el municipio de Texcoco, Edo. de México. Revista Internacional Administración \& Finanzas. Volumen 6. No. 4. Estados Unidos de America (Pp. 51- 72).

Vargas, Gustavo y Rodríguez, Carlos Mario (2016). Oligopolio y estrategias de competencia en el mercado de minoristas en México. Economía Informa. No. 400. México (Pp. 3-23).

Zamudio, Geovanni (2012). El ágora fronteriza, prácticas de consumo e interacción simbólica en un mercado sobreruedas de Tijuana. Tesis de Maestría en Estudios Culturales. México, El Colegio de la Frontera Norte. [En línea]. Disponible en: https://www.colef.mx/posgrado/wpcontent/uploads/2014/03/TESIS-Zamudio-Santos-Geovanni.pdf. Consulta: 02/9/2016. 